\title{
Deuterium Quadrupole Coupling Constants of Deuterohalogenoacetylenes
}

\author{
N. Heineking, M. Andolfatto, C. Kruse, W. Eberstein, and H. Dreizler \\ Institut für Physikalische Chemie der Christian-Albrechts-Universität, Kiel
}

Z. Naturforsch. 43a, 755-757 (1988); received July 9, 1988

\begin{abstract}
Employing the high resolution of microwave Fourier transform spectroscopy, we investigated the lowest rotational transitions of fluoro-, bromo-, and iodoacetylene-d. Along with the rotational, centrifugal distortion, halogen nuclear quadrupole, and halogen spin-rotation coupling constants, we determined the deuterium quadrupole coupling constants of bromo- and iodoacetylene-d. For fluoroacetylene-d, we redetermined the deuterium nuclear quadrupole coupling constants with higher accuracy.
\end{abstract}

\section{Introduction}

Because of the small quadrupole moment and nuclear charge of the deuterium nucleus, coupling effects in the rotational spectra of linear molecules containing deuterium are only measurable in low- $J$ transitions. Even then, the small splittings often lead to partial overlaps of different hyperfine structure components. Therefore, the analysis of such measurements has to be carried out very carefully. In this work, we employed our recently developed fit procedures [1]. The measurements were performed with our microwave Fourier transform (MWFT) spectrometers in $J$-band (5.3-8.0 GHz) [2] and Ku-band (12.4-18.0 $\mathrm{GHz})$ [3].

For chloro(35)acetylene-d, a very accurate value for the deuterium quadrupole coupling constant has been given by Tack and Kukolich [4]. For fluoroacetylene-d (DCCF), only less reliable data are available [5]. We therefore reinvestigated the $J=1-0$ rotational transition of deuterofluoroacetylene. In the case of deuterobromoacetylene (DCCBr), we recorded the $J=1-0$ and part of the $J=2-1$ rotational transitions of both bromine isotopomers. In addition, we detected the $J=1-0$ and $J=3-2$ rotational transitions of deuteroiodoacetylene (DCCI).

\section{Experimental}

a) Normal fluoroacetylene was prepared by dehalogenation of 1,2-dibromo-fluoroethylene with magnesium in tetrahydrofurane [6]. Deuteration was per-

Reprint requests to Prof. Dr. H. Dreizler, Institut für Physikalische Chemie der Christian-Albrechts-Universität zu Kiel, Ludewig-Meyn-Str. 8, D-2300 Kiel. formed by treating the product with an excess of deuterium oxide. The product of unknown isotopic contents was distilled off at low pressure and temperature.

We performed several recordings of the $J=1-0$ transition in $\mathrm{Ku}$-band under different experimental conditions. No additional splittings due to magnetic coupling of the fluorine nucleus $(I=1 / 2)$ could be observed. All measurements were performed at temperatures of $225-235 \mathrm{~K}$ and pressures of $0.1-0.2 \mathrm{~Pa}$ (0.7-1.5 mTorr).

b) Normal bromoacetylene was prepared by dehydrobromination of 1,2-dibromoethylene (purchased from Aldrich Chemie, Steinheim) with molten potassium hydroxide [7]. Deuteration was performed as described above.

Because of the large quadrupole moment of the bromine nuclei, the molecular rotation angular momentum couples strongly with the bromine nuclear spin $(I=3 / 2$ for both isotopes) to give an "intermediate" angular momentum $F_{1}$. The resulting $F_{1}$-components are further split by coupling of the deuterium spin, so that each rotational transition consists of several narrow multiplets with different $F_{1}$ quantum numbers. The multiplet patterns vary strongly with $F_{1}$. We analysed the $J=1-0$ transitions (in $J$-band) and some of the $F_{1}$-multiplets of the $J=2-1$ transitions (in $\mathrm{Ku}$-band) of both bromine isotopomers.

c) Deuteroiodoacetylene was prepared by first producing dideuteroacetylene from calcium carbide and deuterium oxide. This gas was then led through an alcaline solution of iodine in deuterium oxide and the substitution product trapped by cooling the exhaust gases to $-80^{\circ} \mathrm{C}[8]$. 
Because the rotational spectrum of deuteroiodoacetylene had not yet been assigned, we first searched for the carbon-13 isotopic satellites in the spectrum of normal iodoacetylene in order to refine the assumed structure of DCCI. After these lines had been found, the respective transitions of the deuterated species could easily be assigned (the structure determination thus possible will be published elsewhere). We recorded the $J=1-0$ transition in $J$-band and one $F_{1}$-group of the $J=3-2$ transition in Ku-band.

\section{Deuterium Quadrupole Coupling in the Spectrum of DCCF}

When only the deuterium nuclear spin $(I=1)$ couples to the molecular rotation, the $J=1$ rotational energy level splits into three sublevels with $F$ quantum numbers $0,1,2$ ( $F$ for overall angular momentum). Their first order energies are $(B=$ rotational constant, $D_{J}=$ centrifugal distortion constant, $e Q q=$ quadrupole coupling constant, $c_{I}(\mathrm{D})=$ spin-rotation coupling constant of deuterium)

$$
\begin{aligned}
& E / h(F=2)=2 B-4 D_{J}-0.05 e Q q+c_{I}(\mathrm{D}), \\
& E / h(F=1)=2 B-4 D_{J}+0.25 e Q q-c_{I}(\mathrm{D}), \\
& E / h(F=0)=2 B-4 D_{J}-0.50 e Q q-2 c_{I}(\mathrm{D}) .
\end{aligned}
$$

The $J=0$ rotational level does not split, so that the transition frequencies $v_{i}$ are expressed by

$$
h v_{i}=E(F=i, J=1)-E(F=1, J=0) .
$$

To eliminate the rotational, centrifugal distortion, and spin-rotation coupling constant, we calculated the linear combination

$$
3 v_{1}-2 v_{0}-v_{2}=1.8 e Q q .
$$

We evaluated the $v_{i}$ as arithmetic mean values out of four recordings. This calculation also yielded standard errors of the $v_{i}$ and, via error propagation, the uncertainty of $e Q q$. It was not possible to determine the spin-rotation coupling constants of deuterium and fluorine. The experimental frequencies and derived mean values as well as the derived deuterium quadrupole coupling constant are given in Table 1 .

\section{Coupling Effects in the Spectrum of DCCBr}

The strong coupling of the bromine nuclei perturbs the rotational energy levels, so that a first order treat-
Table 1. Experimental frequencies of the hyperfine components of the $J=1-0$ rotational transition of deuterofluoroacetylene.

\begin{tabular}{llll}
\hline & $\begin{array}{l}F=2-1 \\
\mathrm{MHz}\end{array}$ & $\begin{array}{l}F=1-1 \\
\mathrm{MHz}\end{array}$ & $\begin{array}{l}F=0-1 \\
\mathrm{MHz}\end{array}$ \\
\hline $\begin{array}{l}\text { First recording 17472.0349 } \\
\text { (weighted twice) }\end{array}$ & 174.72 .0969 & 17471.9431 \\
2nd recording & 17472.0353 & 17472.0974 & 17471.9417 \\
3rd recording & 17472.0352 & 17472.0971 & 17471.9441 \\
4th recording & 17472.0353 & 17472.0971 & 17471.9440 \\
Mean values & $17472.0351(2)$ & $17472.0971(2)$ & $17471.9432(10)$
\end{tabular}

Quadrupole coupling constant $e Q q=205.4(12) \mathrm{kHz}$.

Table 2. Experimental frequencies and derived molecular constants of deuterobromo(79)acetylene and deuterobromo (81)acetylene. $-* \Delta v=$ observed minus calculated frequency.

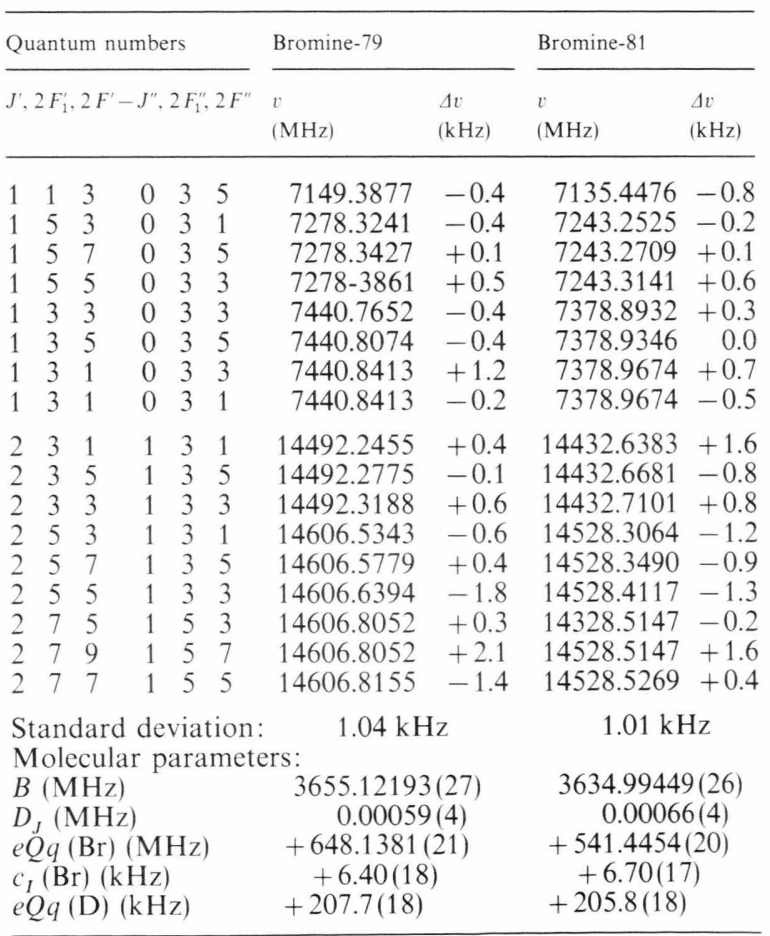

ment is no longer sufficient. Instead, the complete Hamiltonian [9] has to be set up and the energy matrix must be diagonalized. The $J=0$ level remains unsplit $\left(F_{1}=3 / 2\right)$, while the $J=1$ level is split into three $F_{1}$-sublevels $\left(F_{1}=1 / 2,3 / 2,5 / 3\right)$, and the $J=2$ level into four $\left(F_{1}=1 / 2,3 / 2,5 / 2,7 / 2\right)$. Magnetic dipole interaction with the bromine nuclear magnetic moment has to be considered at this step of the analysis. The 
Table 3. Experimental frequencies and derived molecular constants of deuteroiodoacetylene. Sign of $c_{I}(\mathrm{I})$ as defined in [9].

\begin{tabular}{|c|c|c|c|c|c|c|c|}
\hline$J$, & $2 F_{1}$ & $2 F$ & $-J^{\prime \prime}$ & $2 F_{1}^{\prime}$ & $2 F^{\prime \prime}$ & $v(\mathrm{MHz})$ & $\Delta v(\mathrm{kHz})$ \\
\hline 1 & 5 & 5 & 0 & 5 & 5 & 5466.3938 & +0.6 \\
\hline 1 & 5 & 7 & 0 & 5 & 7 & 5466.4407 & -0.8 \\
\hline 1 & 5 & 3 & 0 & 5 & 3 & 5466.4625 & +0.2 \\
\hline 1 & 7 & 5 & 0 & 5 & 3 & 5938.2963 & -1.2 \\
\hline 1 & 7 & 9 & 0 & 5 & 7 & 5938.3124 & +1.9 \\
\hline 1 & 7 & 7 & 0 & 5 & 5 & 5938.3522 & -0.7 \\
\hline 1 & 3 & 5 & 0 & 5 & 7 & 6145.3081 & -0.1 \\
\hline 1 & 3 & 3 & 0 & 5 & 5 & 6145.3210 & +0.1 \\
\hline 3 & 5 & 3 & 2 & 3 & 1 & 17288.6158 & -0.1 \\
\hline 3 & 5 & 7 & 2 & 3 & 5 & 17288.6446 & -0.9 \\
\hline 3 & 5 & 5 & 2 & 3 & 3 & 17288.6828 & +1.0 \\
\hline \multirow{6}{*}{\multicolumn{6}{|c|}{$\begin{array}{l}\text { Standard deviation: } \\
\text { Molecular parameters: } \\
B(\mathrm{MHz}) \\
D_{J}(\mathrm{MHz}) \\
e Q q(\mathrm{I})(\mathrm{MHz}) \\
c_{I}(\mathrm{I})(\mathrm{kHz}) \\
e Q q(\mathrm{D})(\mathrm{kHz})\end{array}$}} & \multicolumn{2}{|l|}{$1.19 \mathrm{kHz}$} \\
\hline & & & & & & \multicolumn{2}{|l|}{$2910.21394(24)$} \\
\hline & & & & & & \multicolumn{2}{|l|}{$0.00040(2)$} \\
\hline & & & & & & \multicolumn{2}{|l|}{$-2254.1785(34)$} \\
\hline & & & & & & \multicolumn{2}{|l|}{$+8.55(18)$} \\
\hline & & & & & & $+209.5(32)$ & \\
\hline
\end{tabular}

Table 4. Comparison of deuterium quadrupole coupling constants of the deuterohalogenoacetylenes (in $\mathrm{kHz}$ ).

\begin{tabular}{lll}
\hline & This work & Older data \\
\hline DCCF & $205.4(12)$ & $212(10)[3]$ \\
DCC $^{35} \mathrm{Cl}$ & - & $208.5(15)[2]$ \\
DCC $^{79} \mathrm{Br}$ & $207.7(18)$ & - \\
DCC $^{81} \mathrm{Br}$ & $206.6(18)$ & - \\
DCCI $^{\mathrm{D}}$ & $209.5(32)$ & - \\
\hline
\end{tabular}

$F_{1}$-sublevels are further split by the deuterium quadrupole coupling. Employing our programme SYM 2QS [10] we analysed the three $F_{1}$-groups of the $J=1-0$ transitions and the three most suitable $F_{1}$-multiplets $\left(F_{1}=7 / 2-5 / 2,5 / 2-3 / 2,3 / 2-3 / 2\right)$ of the $J=2-1$ transitions. From the frequencies of $2 \cdot 17$ recorded hyperfine components, five parameters $\left(B, D_{J}, e Q q(\mathrm{Br})\right.$,

[1] J. Haekel and H. Mäder, Z. Naturforsch. 43a, 203 (1988).

[2] G. Bestmann, H. Dreizler, E Fliege, and W. Stahl, J. Mol. Struct. 97, 215 (1983).

[3] G. Bestmann and H. Dreizler, Z. Naturforsch. 37a, 58 (1982).

[4] L. M. Tack and S. G. Kukolich, J. Mol. Spectrosc. 94, 95 (1982).

[5] V. W. Weiss and W. H. Flygare, J. Chem. Phys. 45, 8 (1966) $e Q q(\mathrm{D})$, and $\left.c_{I}(\mathrm{Br})\right)$ for each isotopomer were determined by simultaneous least squares fit (the centrifugal distortion constant was only included for completeness and is not well determined because of the low- $J$ transitions involved in the analysis). The standard deviation was one kilocycle/sec in both cases. Experimental frequencies and derived data are given in Table 2.

\section{Coupling Effects in the Spectrum of DCCI}

Because of the iodine spin of $5 / 2$, the hyperfine patterns of the rotational transitions are more complicated than those of $\mathrm{DCCBr}$, and the $F_{1}$-multiplets are narrower. Furthermore, the achievable signal intensities are lower due to the very small dipole moment of DCCI. Nevertheless, all three $F_{1}$-groups of the $J=1-0$ rotational transition (in $J$-band) and one $\left(F_{1}=5 / 2-3 / 2\right)$ of the $J=3-2$ transition (in Ku-band) could be recorded and at least partially resolved. From the frequencies of 11 hyperfine components, five molecular parameters (same as above) were determined. The accuracy of these is worse than those of the other investigated molecules but still satisfactory. The experimental frequencies and the derived parameters are presented in Table 3. A compilation of all determined deuterium quadrupole coupling constants, along with those taken from other authors, is given in Table 4. The differences are small, i.e., hardly beyond the error limits, and no systematic variation is obvious.

\section{Acknowledgements}

We thank the members of our group for help and discussions. Funds provided by the Deutsche Forschungsgemeinschaft, the Fonds der Chemie, and the Land Schleswig-Holstein are gratefully acknowledged.

[6] H. G. Viehe and E. Franchimont, Ber. 95, 319 (1962).

[7] W. Oppenheim and L. M. Shorr, Israel J. Chem. 2, 121 (1964).

[8] E. Schäfer and J. J. Christiansen, J. Mol. Struct. 97, 101 (1983).

[9] W. Gordy and R. L. Cook, Microwave Molecular Spectra, 3rd Ed., Chapter XV, John Wiley, New York 1984.

[10] Authors: B. Kleibömer and J. Gripp, Kiel. 